\title{
New Regime of Thomson Scattering: Probing Dense Plasmas with X-Ray Lasers
}

H.A. Baldis, J. Dunn, M.E. Foord, W. Rozmus, C. Andersen and $R$. Shepherd

This article was submitted to

$7^{\text {th }}$ International Conference on X-Ray Lasers

Saint Malo, France

June 18-23, 2000

U.S. Department of Energy

\section{August 30, 2000}

Lawrence

Livermore

National

Laboratory 


\section{DISCLAIMER}

This document was prepared as an account of work sponsored by an agency of the United States Government. Neither the United States Government nor the University of California nor any of their employees, makes any warranty, express or implied, or assumes any legal liability or responsibility for the accuracy, completeness, or usefulness of any information, apparatus, product, or process disclosed, or represents that its use would not infringe privately owned rights. Reference herein to any specific commercial product, process, or service by trade name, trademark, manufacturer, or otherwise, does not necessarily constitute or imply its endorsement, recommendation, or favoring by the United States Government or the University of California. The views and opinions of authors expressed herein do not necessarily state or reflect those of the United States Government or the University of California, and shall not be used for advertising or product endorsement purposes.

This is a preprint of a paper intended for publication in a journal or proceedings. Since changes may be made before publication, this preprint is made available with the understanding that it will not be cited or reproduced without the permission of the author.

This report has been reproduced directly from the best available copy.

Available to DOE and DOE contractors from the

Office of Scientific and Technical Information

P.O. Box 62, Oak Ridge, TN 37831

Prices available from (423) 576-8401

http:/ / apollo.osti.gov/bridge/

Available to the public from the National Technical Information Service

U.S. Department of Commerce 5285 Port Royal Rd., Springfield, VA 22161 http://www.ntis.gov/

OR

Lawrence Livermore National Laboratory Technical Information Department's Digital Library http://www.llnl.gov/tid/Library.html 
UCRL-JC-140304

\title{
New Regime of Thomson Scattering: \\ Probing Dense Plasmas with X-Ray Lasers
}

\author{
H.A. Baldis ${ }^{1,2}$, J. Dunn ${ }^{3}$, M. E. Foord ${ }^{3}$, W. Rozmus 4,1 , C. Andersen ${ }^{2}$, R. Shepherd 3 \\ IInstitute for Laser Science and Applications (ILSA), Lawrence Livermore National Laboratory, \\ POB 808, Livermore CA 94550, USA \\ 2 Department of Applied Sciences, University of California, Davis, CA 95616 \\ ${ }^{3}$ Lawrence Livermore National Laboratory, POB 808, Livermore CA 94550, USA \\ 4 Theoretical Physics Institute, Department of Physics, University of Alberta, \\ Edmonton, Alberta T6G 2J1, Canada
}




\begin{abstract}
In this paper we demonstrate through calculations and theoretical analysis the first application of a x-ray laser for probing hot, high-density plasmas $\left(n_{e} \geq 10^{23} \mathrm{~cm}^{-3}\right)$ using a Ni-like transient collisional excitation $x$-ray laser as a probe. Theoretical predictions are used to diagnose the electron temperature in short pulse $(500 \mathrm{fs})$ laser produced plasmas. The threshold power of the $\mathrm{x}$-ray probe is estimated by comparing theoretical scattering levels with plasma thermal emission. The necessary spectral resolution of the instrument sufficient for resolving electron temperature is given.
\end{abstract}

There has been much progress recently reported on laser-driven soft $\mathrm{x}$-ray laser systems capable of producing megawatts of stimulated emission in the range of $100 \AA$ - $200 \AA$ from Ni-like collisional excitation schemes [1-4]. This opens the door to many exciting opportunities for many applications including microscopy [5], x-radiography [6], and for use in probing high-density plasmas $[7,8]$ with lower energy $\mathrm{x}$-ray laser pump sources. Longer wavelength lasers are typically employed for many plasma diagnostic applications such as interferometry, Thomson scattering (TS) and Faraday rotation, but are limited to lower densities, typically in the range $10^{20}-10^{21} \mathrm{~cm}^{-3}$ for visible-UV light. X-ray lasers therefore offer the exciting possibility of probing orders of magnitude higher densities than previously reached.

Thomson scattering from stable density fluctuations is a powerful diagnostic used to measure local plasma parameters, distribution functions and transport processes [9]. In laser produced plasmas TS has become a standard technique for studying enhanced fluctuations and related instabilities [10]. In this paper we demonstrate through calculations and theoretical analysis the first application of an existing $\mathrm{x}$-ray laser for probing high-density plasmas. We find necessary conditions for the probe intensity, spectral resolution of the spectrometer and sensitivity of the scattered $\mathrm{x}$-ray detection system for the measurement of electron temperature in short pulse laser-produced plasmas. Hard $\mathrm{x}$-ray from laser produced plasmas has been used [11] and proposed [12] for the diagnostic of dense warm matter.

We first discuss the general application of TS as a probe of hot [13] dense plasmas. The goal of this discussion is to demonstrate the feasibility of such a measurement with an existing $x$-ray laser [2] as well as to illustrate several general issues related to the applications of TS as plasma diagnostic at high particle densities. TS of the $\mathrm{x}$-ray laser beam allow a direct insight into a dynamical electron density correlation function. The novelty of our study is in the examination of this function for plasma conditions previously inaccessible to TS experiments.

Consider a geometry of the measurement where $\theta$ is the angle between the direction of the probe propagation, along $\boldsymbol{k}_{\boldsymbol{0}}$ and the direction of the scattered light detection, parallel to $\boldsymbol{k}_{1}$. The angle $\theta$ defines the $k$-vector in a plasma, $k=\left|k_{0}-k_{1}\right|\left[k=2 k_{0} \sin (\theta / 2)\right]$, of the spectral component of an electron density correlation function contributing to the TS cross-section. The probed $k$-vector and parameter $\alpha=1 / k \lambda_{D}$ define two regimes of the incoherent TS: collective for $\alpha>1$, where the crosssection is dominated by electrostatic plasma mode resonance and single particle for $\alpha<1$, where single particle distribution function defines form of the electron density correlation's.

The range of plasma parameters in the planned experiment $\left(T_{e} \approx 50 \mathrm{eV}, n_{e} \geq 10^{23} \mathrm{~cm}^{-3}\right)$ and the wavelength of the Thomson probe $(\lambda=147 \AA$ ) lead to values of $\alpha$ larger than one $(\alpha>1)$ for all scattering angles $\theta$, thus allowing us to limit the discussion to a case of the collective TS. We will 
examine in details the low frequency part of the TS cross-section, which is dominated by ion-acoustic wave resonance. From the position of two maxima separated by two ion acoustic frequencies one can accurately deduce electron plasma temperature.

Our ability to extract spectral properties of an electron density dynamical correlation function, such as for example the location of an ion-acoustic resonance, requires the large degree of spatial and temporal coherence of the probe. The $\mathrm{x}$-ray laser [2] produces a beam, which is characterized by the coherence lengths on the order of $220 \mu \mathrm{m}$ in the longitudinal direction and approximately $10 \mu \mathrm{m}$ in the transverse direction. Both values are much longer than correlation lengths of ion acoustic fluctuations produced in the plasma.

Finally, laboratory plasmas at high densities display temporal and spatial variations. Related inhomogeneities of plasma parameters should be included into theoretical expressions for the TS cross-section together with the effect of a finite spectrometer resolution. Physical properties of the incoherent TS, discussed so far can be described by the following expression [14] for the Thomson scattered power, $P_{s}$, per solid angle, $\Delta \Omega$, the spectral width, $\Delta \lambda$, and the scattering volume, $V$ :

$$
\frac{P_{s}}{\Delta \Omega \Delta \lambda V}=I_{p} \frac{1}{4 \pi}\left(1+\cos ^{2} \theta\right)\left(\frac{e^{2}}{m_{e} c^{2}}\right)^{2} \frac{2 \pi c}{\lambda^{2}} \int d \lambda_{1} G\left(\lambda-\lambda_{1}\right) \frac{1}{2 L} \int_{-L}^{L} d x S\left(k, \omega\left[\lambda_{1}\right] ; x\right) n_{e}(x),(1)
$$

where $I_{p}$ is the x-ray probe intensity, whose coherence within the scattering volume, $V$, has been assumed in the derivation of this expression. The function $G(\lambda)$ describes spectral response function of the instrument. $G(\lambda)$ has been convoluted with the spatially averaged dynamical form factor $S(k, \omega)$, i.e. Fourier transformed electron density correlation function. The derivation of Eq. (1) has taken into account a specific geometry of the planned experiment, where the gradients of temperature and electron density are along the $x$-direction, which is also Thomson probe propagation direction. $2 L$ is the linear dimension of the scattering volume. The spatial scales of plasma parameter variations are much longer than characteristic correlation lengths, allowing separation of scales and local calculations of the dynamical form factor.

The standard expression for the dynamical form factor can be derived from the collisionless theory of plasma fluctuations [15]

$$
S(\boldsymbol{k}, \omega)=\frac{2 \pi}{k}\left|1-\frac{\chi^{e}(\boldsymbol{k}, \omega)}{\varepsilon(\boldsymbol{k}, \omega)}\right|^{2} \bar{f}^{e}(v=\omega / k)+\left|\frac{\chi^{e}(\boldsymbol{k}, \omega)}{\varepsilon(k, \omega)}\right|^{2} z \bar{f}^{i}(v=\omega / k),
$$

where $\bar{f}^{\alpha}(v=\omega / k), \alpha=e, i$, is the particle distribution function integrated with respect to velocity components which are transverse to the direction of $k$. The distribution functions are evaluated at the resonance velocity $\omega / k$. The dielectric response function, $\varepsilon(k, \omega)=1+\sum_{\alpha=e, i} \chi^{\alpha}(k, \omega)$, is calculated using the usual collisionless forms of plasma susceptibility functions $\chi^{\alpha}$ [15]. Important generalization of the expression for $S(k, \omega)$ (2) in inhomogeneous plasmas corresponds to the evaluation of (2) locally at different positions using the local Maxwellian distribution function [14].

High electron density allows examination of the plasma response under conditions, which have been inaccessible in the past to the TS experiments. For example, conditions of a planned experiment $\left(T_{e} \approx 50 \mathrm{eV}, n_{e} \geq 10^{23} \mathrm{~cm}^{-3}, \theta=20^{\circ}\right)$ lead to $k \lambda_{e i} \approx 0.11$, where $\lambda_{e i}$ is the electron-ion mean-free-path. For this value of the wave-vector $k$, the dynamical form factor $S(k, \omega)$ must be derived from the kinetic theory including weak collisional effects. [16].

In view of the limited power output of the $\mathrm{x}$-ray laser we first apply the TS cross-section expression (1) in threshold calculations, where by comparison with plasma thermal emission levels we find the necessary intensity of the x-ray probe, $I_{p}$. For this threshold value of $I_{p}$ Thomson scattered signal equals to bremsstrahlung emission. We use the following expression for the power due to thermal emission $P_{B}$, per solid angle, $\Delta \Omega$, the spectral width, $\Delta \lambda$, and the unit volume in the plasma [17]:

$$
\frac{P_{B}}{\Delta \Omega \Delta \lambda V}=1.510^{31} \frac{Z n_{e}^{2}}{\lambda^{2} T_{e}^{1 / 2}} \overline{g_{f f}} \exp \left(-\frac{1.2410^{4}}{\lambda T_{e}}\right)
$$


where $\overline{g_{f f}}$ is an averaged Gaunt factor close to one for the present plasma parameters. Results of threshold calculations are displayed in Fig. 1, showing several intensity curves on the plane of electron density and temperature. The relevant range of plasma parameters must be located above the threshold intensity curve.

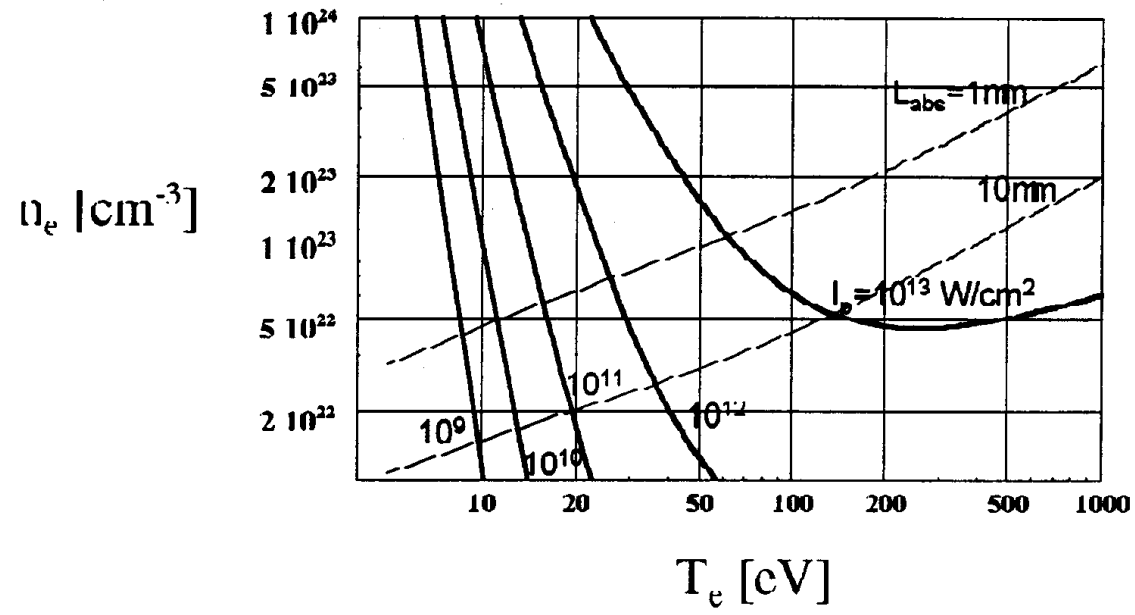

Figure 1: The electron density vs temperature plane. The curves show threshold values of the Thomson probe at $\lambda=147 \AA$ necessary to overcome thermal emission levels at the wavelength of the probe. Lines marked by $\mathrm{L}_{\mathrm{abs}}$ define the collisional absorption length of the $\mathrm{x}$-ray radiation.
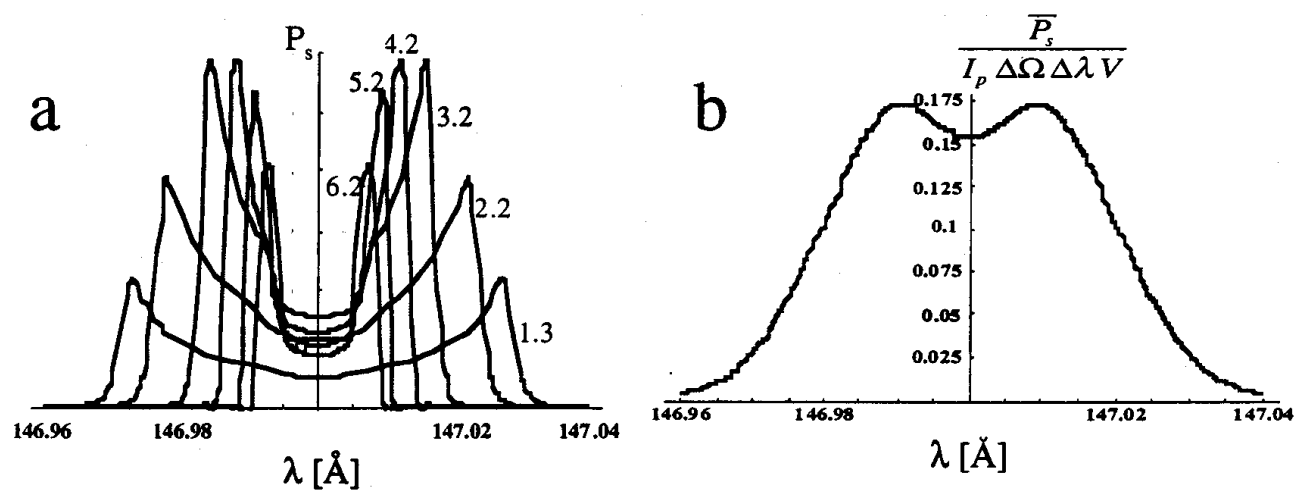

Figure 2: (a) Thomson scattered power calculated from Eq. (1) at different moments in time. The spectral density is averaged in space with the different density and temperature profiles shown in Fig. 2. (b) Time averaged Thomson scattered power convoluted with the instrumental width.

In the planned experiment a x-ray probe is scattered from the high-density plasma produced by subpicosecond laser pulses. Such plasmas are routinely generated within a table top size experimental set-up, providing a unique realization of close to solid density conditions.

We proceed by performing simulations of the laser absorption and hydrodynamics using the code LASNEX [18]. The target consists of a $0.22 \mu \mathrm{m}$ aluminum foil heated by the 500 fs laser pulse of $1 \mathrm{~J}$ energy focussed to the intensity of $10^{16} \mathrm{~W} / \mathrm{cm}^{2}$ in the spot of approximately $100 \mu \mathrm{m}$ diameter. The simulation continues over several picoseconds, including the time of short pulse laser and $x$-ray probe duration. The latter is delayed with respect to plasma forming pulse and lasts for approximately $5 \mathrm{ps}$. The x-ray laser follows the $1 \mu \mathrm{m}$ laser pulse along the same direction with approximately the same spot cross-sectional area in the target. This geometry has been used in the derivation of the Thomson scattered $\mathrm{x}$-ray power (1). 
We now calculate the $\mathrm{x}$-ray laser Thomson scattered spectrum, and discuss the necessary instrumental resolution and sensitivity required for the measurement of electron temperature. Results of hydrodynamic simulations have been applied in the calculations of the spectral density of the scattered power (1). Using the local expression for the dynamical form factor $S(k, \omega)$ we have found spatially averaged profiles of the TS cross-sections at different moments in time and displayed them in Fig. 3a. Early time scattering is characterized by the wide separation of ion-acoustic maxima due to larger temperature, but smaller scattering levels. At these early times, before the foil explosion, the effective scattering volume is smaller, reducing the overall scattered power. Figure $3 \mathrm{~b}$ shows an averaged in time spectral density of the scattered power, which also includes an effect of the function $G(\lambda)(1)$, Gaussian for simplicity, describing the instrumental broadening. The plot displayed in Fig. $3 b$ involves an assumption of the instrumental resolution of $A_{\lambda}=\Delta \lambda / \lambda=10^{-4}$, a Gaussian profile with a $14.7 \mathrm{~m} \AA$ (FWHM). This result shows that with a resolution of $10^{-4}$, the two ionacoustic peaks may be differentiated and thus the electron temperature determined.

In addition to the requirements of high resolving power, the instrumental sensitivity which includes the efficiencies of collection and signal conversion must be sufficient to overcome detector background levels. For the plasma conditions described in Fig. 3 (b), the Thomson scattered x-ray laser power is $\sim 4.4 \times 10^{3} \mathrm{~W} /$ sphere/Å. In order to obtain the high resolving power and narrow spectral coverage required to make these measurements, we consider employing a multilayer-coated grating spectrometer operating at close to normal incidence [19]. Estimates of instrumental sensitivity show that approximately 70 photons are detected within an instrumental resolution channel when the solid angle collection and reflectivity of a large cylindrical optic, entrance slit transmission, multilayer grating reflectivity and typical back-thinned CCD quantum efficiency are included. We find that intensities near $I_{p}=10^{12} \mathrm{~W} / \mathrm{cm}^{2}$ are required to probe these simulated plasmas, close to the current operating regime of the Ni-like transient $\mathrm{x}$-ray laser system $\left(\mathrm{I}=2 \times 10^{11} \mathrm{~W} / \mathrm{cm}^{2}\right)$. We believe that by further optimizing the $\mathrm{x}$-ray laser and target geometry, the required threshold intensities may be achieved.

\section{Acknowledgments}

The authors gratefully acknowledge discussions with Drs. O. L. Landen, D. Riley, V. N. Shlyaptsev, and A. Osterheld. This work was partially supported under the auspices of the U.S. Department of Energy by the Lawrence Livermore National Laboratory under contract No.W-7405-ENG-48. Part of this support was provided through the LLNL Institute for Laser Science and Applications. Research of W. R. has been partially supported by the Natural Science and Engineering Research Council of Canada.

\section{References}

[1] J. Zhang, A.G. MacPhee, J. Nilsen, et al., Phys. Rev. Lett. 78, 3856 (1997).

[2] J. Dunn, Y. Li, A. L. Osterheld, J. Nilsen, et al., Phys. Rev. Lett. 84, 4834 (2000).

[3] S. Sebban, H. Daido, N. Sakaya, et al., Phs. Rev. A 61, 043810 (2000).

[4] C.L.S. Lewis, R. Keenan, A.G. MacPhee, et al., Proc. SPIE - Int. Soc. Opt. Eng. 3776, 292 (1999).

[5] L.B. Da Silva, J.E. Trebes, R. Balhorn, et al., Science 258, 269 (1992).

[6] D.H. Kalantar, M.H. Key, L.B. Da Silva, et al. , Phys. Rev Lett. 76, 3574 (1996).

[7] R. Cauble, L.B. Da Silva, T.W. Barbee,Jr., et al., Phys. Rev. Lett. 74, 3816 (1995).

[8] J. Filevich, K. Kanizay, M.C. Marconi, et al., Opt. Lett. 25, 356 (2000).

[9] B. La Fontaine, H. A. Baldis, D. M. Villeneuve, et al. Phys. Plasmas 1, 2329 (1994). and references therein

[10] S. Depierreux, J. Fuchs, C. Labaune, et al., Phys. Rev. Lett. 84, 2869 (2000). ). and references therein

[11] D. Riley, N. C. Woolsey, D. McSherry, I. Weaver, A. Djaoui, and E. Nardi, Phys. Rev. Lett. 84, 1704 (2000).

[12] O. L. Landen, S. H. Glenzer, R. C. Cauble, R. W. Lee, J. E. Edwards, and J. S. DeGroot, "Warm, Dense Plasma Characterization by X-ray Thomson Scattering", Proceedings of ECLIM, 2000.

[13] Electron density must be small enough for the plasma to be transparent for the Thomson probe. Usually limiting $n_{e}$ to 0.1 of the critical density, $n_{c}$, eliminates refraction and strong absorption. For the $\mathrm{x}$-ray laser wavelength of $147 \AA$, $n_{c}=4.410^{24} \mathrm{~cm}^{-3}$.

[14] W. Rozmus, S. H. Glenzer, K. G. Estabrook, H. A. Baldis, and BJ. MacGowan, ApJS, in press (2000).

[15] J. Sheffield, Plasma Scattering of Electromagnetic Radiation (New York, Academic, 1975).

[16] J. F. Myatt, W. Rozmus, V. Yu. Bychenkov, and V. T. Tikhonchuk, Phys. Rev. E 57, 3383 (1998); J. F. Myatt, PhD Thesis, University of Alberta, unpublished (1997).

[17] Plasma DiagnosticsTechniques, ed. R.H. Huddlestone and S.L. Leonard, pp. 627 (New York, Academic, 1965).

[18] G.B. Zimmerman and W.L. Kruer, Comments Plasma Phys. Controlled Fusion 2, 51 (1975).

[19] J. Seely, C. Brown, Naval Research Laboratory, private communication (1999). 